\title{
Pulsatile control of rotary blood pumps: Does the modulation waveform matter?
}

\author{
Tohid Pirbodaghi, MSc, ${ }^{\mathrm{a}}$ Shannon Axiak, DVM, ${ }^{\mathrm{b}}$ Alberto Weber, MD, ${ }^{\mathrm{c}}$ Thomas Gempp, $\mathrm{PhD},{ }^{\mathrm{d}}$ and \\ Stijn Vandenberghe, $\mathrm{PhD}^{\mathrm{a}}$
}

\begin{abstract}
Objective: Mechanical support of a failing heart is typically performed with rotary blood pumps running at constant speed, which results in a limited control on cardiac workload and nonpulsatile hemodynamics. A potential solution to overcome these limitations is to modulate the pump speed to create pulses. This study aims at developing a pulsatile control algorithm for rotary pumps, while investigating its effect on left ventricle unloading and the hemodynamics.
\end{abstract}

\begin{abstract}
Methods: The CentriMag (Levitronix GmbH, Zürich, Switzerland) rotary blood pump was implanted in 5 sheep and cannulated from the ventricular apex to the descending aorta. A modified controller was connected to the pump yielding direct speed control via analog voltage. Pump speed modulation patterns, including sine, saw tooth, triangle, and square waveforms with 2 different phase shifts, were synchronized with heartbeat. Various hemodynamic parameters, such as left ventricular pressure and volume, coronary flow, and arterial pressure, were analyzed to examine the influence of pump support.
\end{abstract}

Results: The pump speed modulation significantly affected left ventricular pressure and volume and arterial pressure, whereas coronary flow was not influenced by pump support mode. Stroke work in the pulsatile modes varied from $69 \%$ to $91 \%$ of baseline value and from $74 \%$ to $96 \%$ of constant speed value. Consequently, cardiac workload can be adjusted to provide relaxation, which may lead to myocardial recovery.

Conclusions: A synchronized pulsing rotary blood pump offers a simple and powerful control modality for heart unloading. This technique provides pulsatile hemodynamics, which is more physiologic than continuous blood flow and may be useful for perfusion of the other organs. (J Thorac Cardiovasc Surg 2012;144:970-7)

For the past decade, ventricular assist devices (VADs) based on rotary blood pumps (RBPs) have been increasingly used for short- and long-term mechanical support of patients with severe heart failure. The nonphysiologic flow characteristics of these devices provide a continuous blood flow (altered slightly by heart contractility) in contrast with the first generation of VADs based on pulsatile pumps ${ }^{1}$ because of the typical use of constant speed in clinically available RBPs. Studies on the effect of continuous flow for longterm support show that nonpulsatile flow increases the incidence of gastrointestinal bleeding ${ }^{2,3}$; in addition, it increases the risk of adverse events, such as peripheral vasculature stiffening and hemorrhagic strokes. ${ }^{4-6}$

From ARTORG Cardiovascular Engineering, ${ }^{a}$ University of Bern, Bern, Switzerland; Department of Veterinary Anesthesia, ${ }^{\mathrm{b}}$ University of Bern, Bern, Switzerland; Department of Cardiac Surgery, ${ }^{\mathrm{c}}$ Inselspital University Hospital, Bern, Switzerland; and Levitronix $\mathrm{GmbH},{ }^{\mathrm{d}}$ Zurich, Switzerland.

Funding: This study was funded with the support of the Swiss National Science Foundation, project 32003B_127443/1. Levitronix GmbH supplied the pump and controller. Bitmedical GmbH and Medtronic Switzerland provided the cannulas and cell saver disposables.

Disclosures: Thomas Gempp is an employee of Levitronix Medical GmbH. All other authors have nothing to disclose with regard to commercial support.

Received for publication Oct 20, 2011; revisions received Dec 29, 2011; accepted for publication Feb 13, 2012; available ahead of print March 13, 2012.

Address for reprints: Tohid Pirbodaghi, MSc, Murtenstrasse 50, Postfach 44, CH3010 Bern, Switzerland (E-mail: pirbodaghi@artorg.unibe.ch).

$0022-5223 / \$ 36.00$

Copyright (c) 2012 by The American Association for Thoracic Surgery

doi:10.1016/j.jtcvs.2012.02.015
A promising application of VADs, in addition to their use in bridge-to-transplantation and destination therapy, is the bridge-to-myocardial recovery. ${ }^{7,8}$ Although the mechanism of functional recovery of a failing heart is not completely understood, cardiac unloading by assist devices has been suggested as a mechanical tool to promote recovery. ${ }^{9,10} \mathrm{We}$ hypothesize that synchronized pump speed modulation would result in better control of cardiac workload to stimulate heart healing.

The current study aims to use different basic waveforms to modulate the speed of a CentriMag RBP (Levitronix $\mathrm{GmbH}$, Zürich, Switzerland) synchronized with the electrocardiogram (ECG) and investigates its effect on left ventricular (LV) unloading and hemodynamics. We have examined saw tooth, triangle, sine, and square waveforms as the pump modulation patterns, each with 2 different phase shifts with respect to cardiac contraction (comparable to copulsation and counterpulsation). The highlight of synchronization and using different waveforms is the possibility of better control of cardiac workload and valuable new insight into the potential ability of rotary pumps for heart recovery.

\section{MATERIALS AND METHODS}

\section{Pulsatile Speed Pattern of Rotary Blood Pump}

The CentriMag RBP is a magnetically levitated centrifugal-flow pump composed of a disposable pump head and reusable motor. The device is based on the bearingless motor technology, which results in minimal 


\author{
Abbreviations and Acronyms \\ $\mathrm{AP}=$ arterial pressure \\ $\mathrm{CF}=$ coronary flow \\ $\mathrm{ECG}=$ electrocardiogram \\ $\mathrm{EDV}=$ end-diastolic volume \\ $\mathrm{krpm}=$ kilo revolutions per minute \\ $\mathrm{LV}=$ left ventricular \\ $\mathrm{PAF}=$ pulmonary artery flow \\ $\mathrm{PF}=$ pump flow \\ $\mathrm{PVA}=$ pressure-volume area \\ $\mathrm{RBP}=$ rotary blood pump \\ $\mathrm{SV}=$ stroke volume \\ $\mathrm{SW}=$ stroke work \\ $\mathrm{VAD}=$ ventricular assist device
}

friction in the blood path and works with direct drive yielding sturdy control over the speed. A Levitronix industrial controller was modified and tuned to run with the pump. This modified proportional-integral controller yields direct speed control via analog voltage and feedback of the actual speed. The custom-made control program, which has QRS detection and phase shift-tracking algorithms, was written in LabVIEW (National Instruments, Austin, Tex) and communicates in real-time via a data acquisition system (c-RIO-9074, National Instruments). This program detects the QRS complexes in the ECG signal using a peak detector based on the Pan-Tompkins algorithm ${ }^{11}$ and then produces the pump control signal in synchrony with the heart rate. The program continuously receives the animal's ECG signal using a patient monitor (model AS/3; Datex-Ohmeda Division, Instrumentarium Corp, Helsinki, Finland). Figure 1, A shows a description of the pump speed commands using different waveforms with 2 phase shifts. All the waveforms have the same mean speed (2 kilo revolutions per minute $[\mathrm{krpm}])$ and amplitude $(1 \mathrm{krpm})$; therefore, regardless of the waveform type, the number of pump impeller revolutions per each heart beat is equal. $\mathrm{krpm}$ is a measure for rotational speed of the pump impeller. Figure $1, B$, presents the actual pump speed demonstrating how well the pump can follow the command speed.

\section{Surgical Preparation and Instrumentation}

The CentriMag RBP was implanted in 5 healthy female sheep (56-83 $\mathrm{kg}$ ). This study was approved by the Swiss Federal Veterinary Office and is in compliance with the Guide for the Care and Use of Laboratory Animals (National Academy of Sciences, 1996). All animals received humane care in compliance with the Guide for the Care and Use of Laboratory Animals. After premedication and induction of anesthesia, the animals were intubated and anesthesia was maintained with isoflurane in oxygen $(1.6 \%)$ and fentanyl $(5-10 \mu \mathrm{g} / \mathrm{kg} / \mathrm{h})$. A left thoracotomy was used to enter the thorax and expose the LV apex and descending aorta (used in lieu of a short and inaccessible ascending aorta). The animals were heparinized to maintain the whole blood activated clotting time greater than 400 seconds. For surgical implantation of pump inflow, LV apical cannulation was performed using a modified $32 \mathrm{~F}(1 \mathrm{~F}=0.33 \mathrm{~mm})$ angled venous cannula (DLP 67532; Medtronic Inc, Minneapolis, Minn), and a $22 \mathrm{~F}$ arterial cannula (EOPA 77522 , Medtronic Inc) was placed in the descending aorta. The cannulation was kept short $(35 \pm 5 \mathrm{~cm})$ to simulate implantable VADs and avoid energy losses inside the inlet/outlet cannula. After pump priming and connection of the cannulas to the inlet/outlet ports, pump operation commenced. Pulmonary artery flow (PAF), coronary flow (CF), and pump flow (PF) were measured using ultrasonic flow probes (Transonic Systems Inc, Ithaca, NY) on the pulmonary artery (24PAU), left main coronary artery (8PAU), and outlet cannula of the pump (9PXL), respectively. LV pressure and volume were
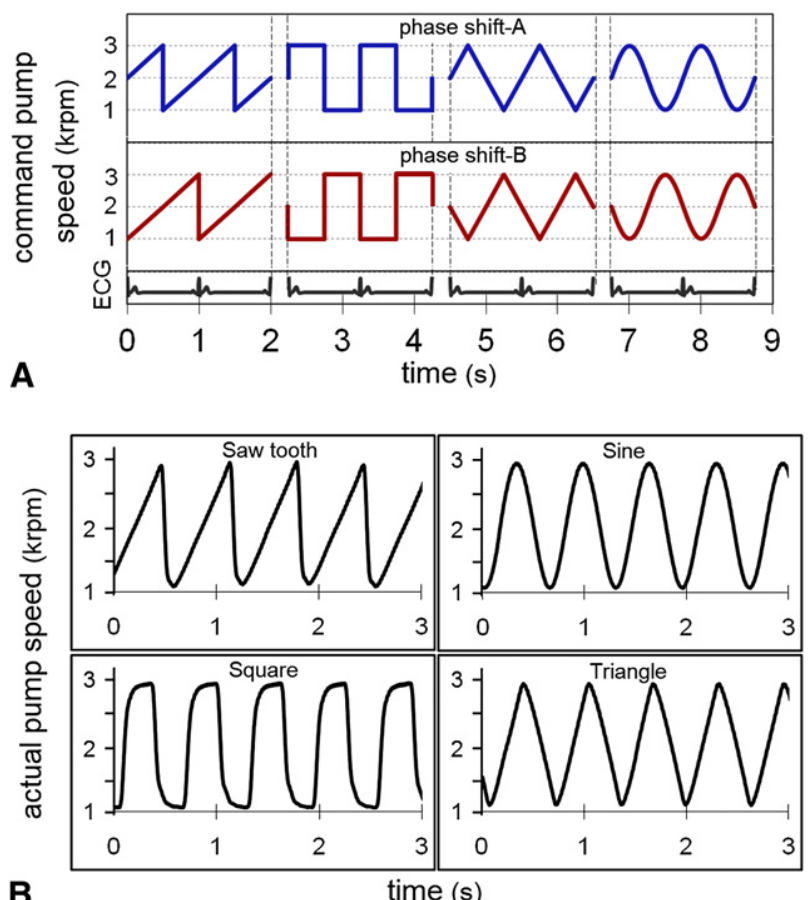

FIGURE 1. A, Various types of waveforms synchronized with electrocardiogram $(E C G)$ as pump speed command. Phase shifts A and B have a 180-degree phase difference. $\mathrm{krpm}$ is a measure for rotational speed of the pump. B, Actual pump speed derived from the motor's Hall sensors.

acquired by a 7F admittance catheter (Scisense Inc, London, Ontario, Canada) in the LV via the right carotid artery. In addition, this catheter includes an extra pressure sensor to obtain arterial pressure (AP) in the ascending aorta. Actual speed of the pump impeller was monitored via an analog output at the pump controller from the motor's Hall sensors. All signals were acquired using iWorx data recorder and LabScribe data acquisition software (iWorx Systems, Inc, Dover, NH) at a sampling rate of $200 \mathrm{~Hz}$. The cRIO9074 was used to generate analog voltages to command the pump speed. Before recording, all pressure sensors were soaked in warm saline and zeroed to atmosphere and calibrated with a PXCAL medical pressure calibrator (Edwards Lifesciences Inc, Irvine, Calif) over their expected range. Flow sensors also were submerged in water baths and zeroed.

\section{Study Protocol}

The study protocol was performed chronologically as follows:

- First, baseline measurements including hemodynamic data (ECG, AP, $\mathrm{LV}$ pressure and volume, $\mathrm{CF}, \mathrm{PF}$, and PAF) were recorded. Baseline is defined as the condition where the pump is switched off and its inlet cannula is clamped.

- Second, a series of LV pressure-volume loops were measured during inferior vena cava occlusion at baseline. Pressure-volume loops during inferior vena cava occlusion were used to derive volume-axis intercept, $\mathrm{V}_{0}$ in the PV plane for LV data.

- Third, 4 different waveforms (sine, square, saw tooth, and triangle) with 2 phase shifts (defined as phase shifts A and B in Figure 1, A) synchronized with the ECG were prescribed for the pump speed (the order of the waveforms was randomized for all sheep).

\section{Statistical Analysis}

One-way analysis of variance was performed to assess the effect of the pump speed on the LV unloading and hemodynamic parameters. In 
TABLE 1. Characteristics of all sheep at baseline

\begin{tabular}{lcccccccccc}
\hline $\begin{array}{c}\text { Sheep } \\
\text { no. }\end{array}$ & $\begin{array}{c}\text { Weight } \\
(\mathbf{k g})\end{array}$ & $\begin{array}{c}\text { HR } \\
\text { (beats/min) }\end{array}$ & $\begin{array}{c}\text { AP } \\
(\mathbf{m m} \mathbf{~ H g})\end{array}$ & $\begin{array}{c}\text { PP } \\
(\mathbf{m m} \mathbf{H g})\end{array}$ & \multicolumn{1}{c}{$\mathbf{S V}(\mathbf{m L})$} & $\mathbf{E D V}(\mathbf{m L})$ & $\begin{array}{c}\text { SW } \\
(\mathbf{m m} \mathbf{H g} / \mathbf{m L})\end{array}$ & $\begin{array}{c}\text { PVA } \\
(\mathbf{m m} \mathbf{H g} / \mathbf{m L})\end{array}$ & $\mathbf{C F}(\mathbf{m L})$ & $\mathbf{P A F}(\mathbf{L} / \mathbf{m i n})$ \\
\hline 1 & 77 & $88 \pm 4.4$ & $55.2 \pm 4.9$ & $27.5 \pm 2.2$ & $58.1 \pm 1.9$ & $86.3 \pm 1.8$ & $4377 \pm 136$ & $4801 \pm 162$ & $471 \pm 12$ & $5.07 \pm 0.23$ \\
2 & 83 & $68 \pm 1.5$ & $51.2 \pm 3.7$ & $38.5 \pm 0.5$ & $60.3 \pm 2.8$ & $138 \pm 3.5$ & $3708 \pm 195$ & $6194 \pm 151$ & - & $5.03 \pm 0.19$ \\
3 & 68 & $71 \pm 0.8$ & $51.1 \pm 4.1$ & $33.4 \pm 1.5$ & $80 \pm 1.6$ & $124 \pm 1.4$ & $4849 \pm 28$ & $6717 \pm 28$ & $173 \pm 18$ & $4.81 \pm 0.34$ \\
4 & 69 & $47 \pm 1$ & $44 \pm 3.5$ & $30.5 \pm 0.9$ & $57.1 \pm 6.2$ & $71.4 \pm 6.8$ & $3294 \pm 227$ & $3801 \pm 227$ & - & $4.25 \pm 0.1$ \\
5 & 56 & $94 \pm 0.7$ & $52.5 \pm 2.9$ & $29.9 \pm 1.1$ & $18 \pm 3.7$ & $81 \pm 5.2$ & $1519 \pm 203$ & $2209 \pm 221$ & $340 \pm 26$ & $4.55 \pm .31$ \\
\hline
\end{tabular}

Values are mean \pm standard deviation, with the average over $\sim 20$ cardiac cycles. $H R$, Heart rate; $A P$, arterial pressure; $P P$, pulse pressure; $S V$, stroke volume; $E D V$, end-diastolic volume; $S W$, stroke work; $P V A$, pressure-volume area; $C F$, coronary flow; $P A F$, pulmonary artery flow.

addition, Tukey's post test was used to compare different pump speed patterns with each other and with the baseline and constant speed groups using GraphPad Prism 5.0 (GraphPad Software, San Diego, Calif).

\section{RESULTS}

Table 1 summarizes the sheep characteristics and energetic and hemodynamic data at baseline. Figure 2 illustrates the definition of stroke work (SW), pressure-volume area (PVA), stroke volume (SV), and end-diastolic volume (EDV). SW corresponds to the energy that LV transfers to blood and PVA is the energy consumed by LV to contract. $\mathrm{SV}$ is the volume of blood pumped from LV with each beat. Moreover, pulse pressure (PP) is the difference between the systolic and diastolic pressures in the aorta. LabScribe software was used to calculate the aforementioned parameters, which are averaged over 20 heart cycles.

The average ventricular pressure-volume loops over 20 heart cycles are shown in Figure 3 for sheep number 1 as a typical example. Compared with the baseline and constant speed, the main effect of the modulated pump speed is the smaller pressure-volume loops. For all the waveforms, the loops show a decrease in EDV, end-systolic volume, and end-systolic pressure. During pump support using the waveforms, the square B and saw tooth A have the smallest and largest pressure-volume loops, respectively.

Figure 4 displays the effect of different waveforms and phase shifts on AP. Because of the pump support using

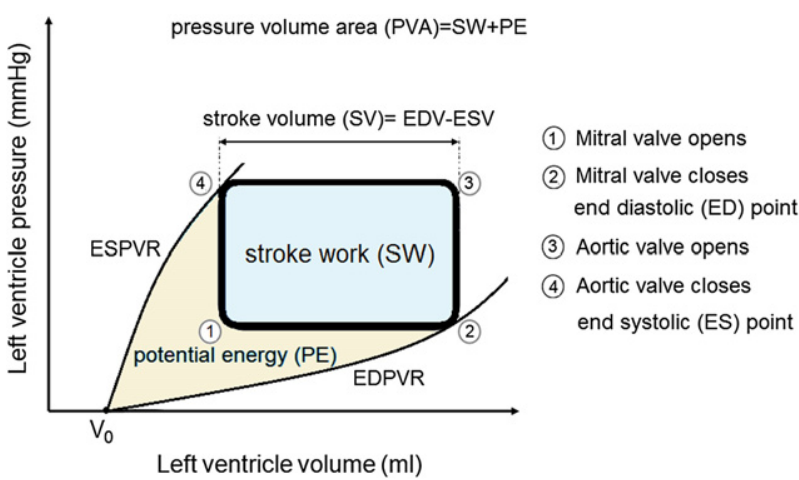

FIGURE 2. Definition of stroke work $(S W)$, pressure volume area $(P V A)$, and stroke volume $(S V)$. $E D V$, End-diastolic volume; $E S V$, end-systolic volume; $E S P V R$, end-systolic pressure volume relation; $E D P V R$, end-diastolic pressure volume relation (V0 is the dead volume of left ventricle). waveforms, minimum AP increases and its maximum value decreases compared with the baseline, whereas the mean value shows only a negligible variation $(53.4 \pm 2.5 \mathrm{~mm}$ $\mathrm{Hg}$ ). For all the waveforms, the PP decreases while phase shift $B(15.1 \pm 2.3 \mathrm{~mm} \mathrm{Hg})$ shows lower values than phase shift A $(19.2 \pm 1.9 \mathrm{~mm} \mathrm{Hg})$. Constant speed support has the lowest $\mathrm{PP}$ value $(7.1 \pm 0.8 \mathrm{~mm} \mathrm{Hg})$. It should be mentioned that there is an insignificant pulsatile AP during constant speed support, whereas for a failing heart all the pulsatility effect of the native heart can be diminished.

In Figure 5, PF patterns are compared (for sheep number 1). Sine $A$ and square $A$ result in back flow $(-0.85 \pm 0.05$ and $-1.18 \pm 0.1 \mathrm{~L} / \mathrm{min}$, respectively) through the pump, whereas for the other pump speed patterns, there is always positive flow. For saw tooth $\mathrm{B}$ and triangle $\mathrm{B}$, the minimum $\mathrm{PF}(2.11 \pm 0.08$ and $2.0 \pm 0.08 \mathrm{~L} / \mathrm{min}$, respectively) increases remarkably compared with the other waveforms. During constant speed (2000 rpm) support, the slight pulsatility in PF is completely caused by the beating heart. By integrating the area under the PF rate vs time graph, the blood volume flowing back into the $\mathrm{LV}$ per beat can be quantified. For all animal data, Table 2 presents the amount of back flow per each heart beat for different pump speed patterns. For the other pump speed patterns, which are not shown in Table 2, there is no backflow through the pump.

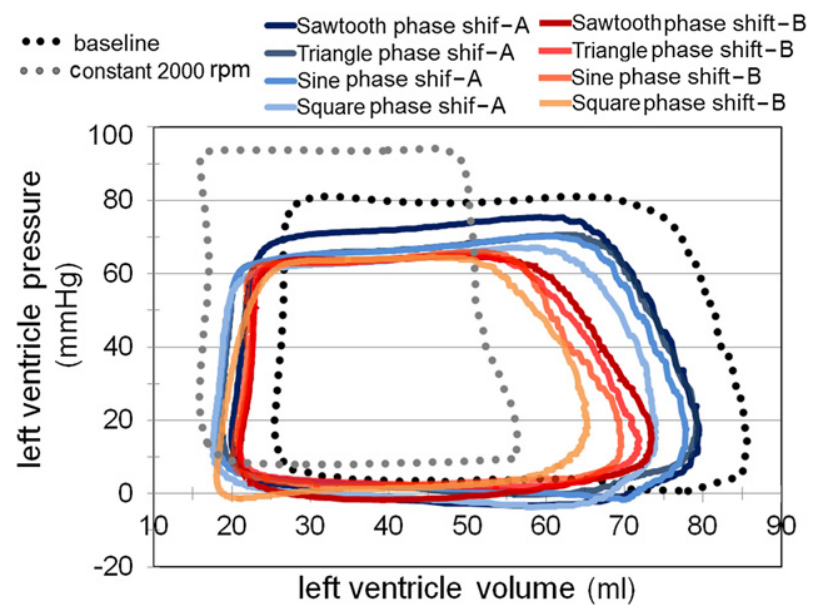

FIGURE 3. Variation of pressure-volume loops due to different pump speed patterns in sheep number 1 as a typical example. 


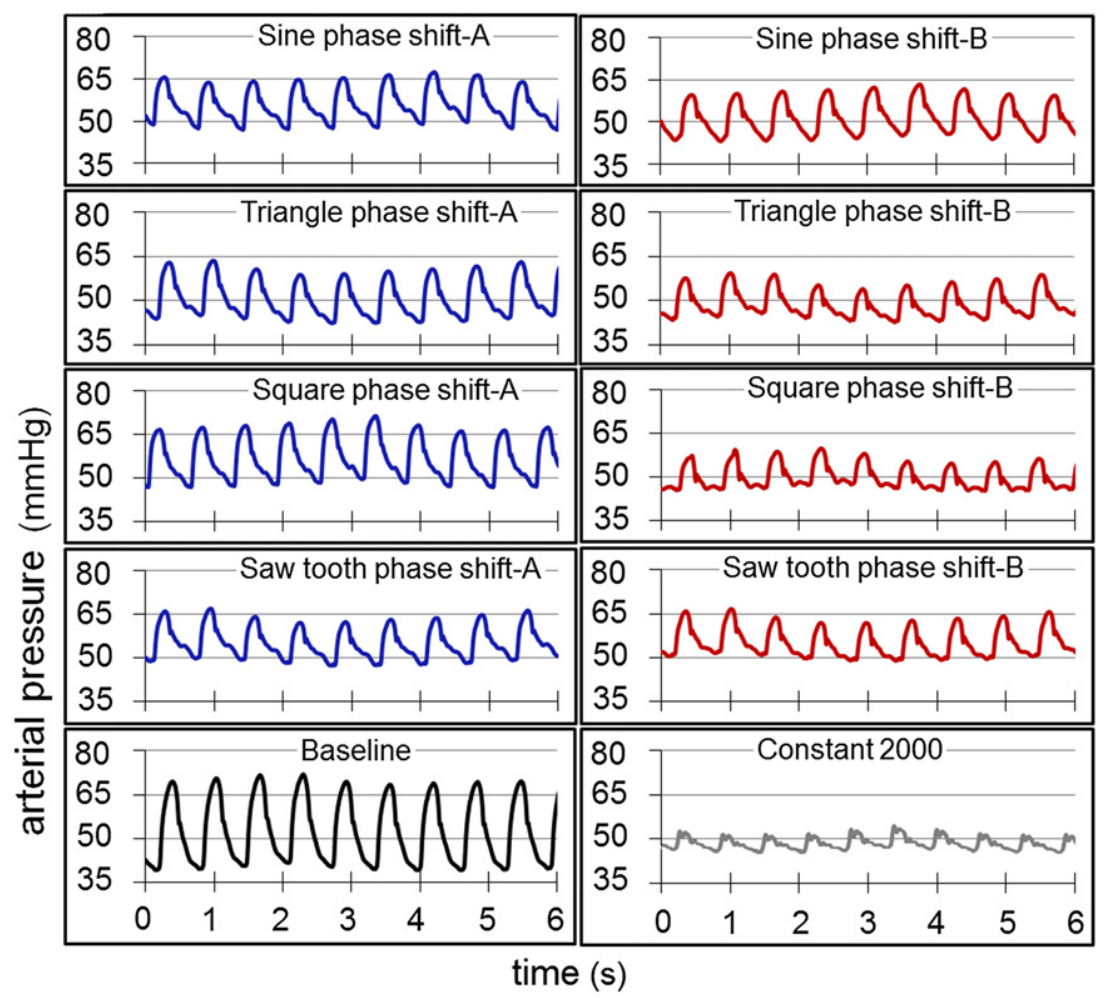

FIGURE 4. Variation of arterial pressure due to different pump speed patterns.

Figure 6 illustrates the CF patterns (for sheep number 1). The flow pattern in the coronary artery is altered slightly by pump support compared with the baseline, although showing almost no difference when the different waveforms are applied. Furthermore, minimum $(-36.6 \pm 20.1 \mathrm{~mL} / \mathrm{min})$, mean $(366.1 \pm 23.5 \mathrm{~mL} / \mathrm{min})$, and maximum $(685.1 \pm$ $29.8 \mathrm{~mL} / \mathrm{min}$ ) CFs show only a minor variation due to speed modulation of the pump.

The effect of different waveforms on different hemodynamic and energetic parameters is depicted in Figure 7. The values are the average of all animal data and have been nondimensionalized by their respective baseline values. To assess the unloading of the heart because of different waveforms, Figure 7, $A$ and $B$, compare SW $(P=.0001)$ and PVA $(P=.004)$, indicating phase shift $\mathrm{B}$ has lower values than phase shift A. Square B has the smallest SW ( $69 \%$ of the baseline value and $74 \%$ of the constant speed value) and PVA ( $68 \%$ of the baseline value and $70 \%$ of the constant speed value). SW can be controlled between $69 \%$ and $91 \%$ (saw tooth $\mathrm{A}$ ) of the baseline value and from $74 \%$ to $96 \%$ of the constant speed value, thereby controlling the heart load by only changing the pump speed pattern; meanwhile, the mean speed and amplitude remain the same. In combination with the trends in PVA, square B leads to slightly better unloading of the LV.

Figure 7, $C$ and $D$, show the effects of different waveforms on SV and EDV, respectively. For all the waveforms,
$\mathrm{SV}(P=.006)$ and $\mathrm{EDV}(P=.004)$ decrease compared with the baseline and phase shift $B$ has lower values than phase shift A; however, within one phase shift, no significant differences are found between the waveforms. Saw tooth B has the smallest SV $(83 \%$ of the baseline value and $106 \%$ of the constant speed value) and EDV ( $81 \%$ of the baseline value and $97 \%$ of the constant speed value).

The AP and PP are illustrated in Figure 7, $E$ and $F$. The variation of AP due to waveform differences is negligible and statistically insignificant $(P=.37)$, whereas PP is remarkably sensitive to different pump speed patterns $(P=.0001)$. The PP decreases from $31.9 \pm 3.7 \mathrm{~mm} \mathrm{Hg}$ at baseline to $18.8 \pm 4.2 \mathrm{~mm} \mathrm{Hg}(59 \%$ of the baseline value and $138 \%$ of the constant speed value) at square B, and constant speed $(2000 \mathrm{rpm})$ support has the lowest value $(7.3 \pm$ $2.3 \mathrm{~mm} \mathrm{Hg}$ ).

Figure 7, $G$ and $H$, present the effect of waveforms on $\mathrm{CF}$ and PAF, correspondingly. CF $(P=.52)$ and $\operatorname{PAF}(P=.46)$ are almost at constant level, and the effect of different waveforms is not statistically significant.

Figure 8, $A$ and $B$, shows the variation on the PF and minimum PF. Sine A has the lowest $(3.15 \pm 0.21 \mathrm{~L} / \mathrm{min})$, and square $\mathrm{B}$ has the largest $(4.10 \pm 0.19 \mathrm{~L} / \mathrm{min}) \mathrm{PF}$ $(P=.0001)$. In addition, it is important to notice that although all the waveforms have the same mean speed and amplitude, squares $\mathrm{A}(-1.36 \pm 0.18 \mathrm{~L} / \mathrm{min})$ and $\mathrm{B}$ $(-0.33 \pm 0.94 \mathrm{~L} / \mathrm{min})$, triangle A $(-0.15 \pm 0.26 \mathrm{~L} / \mathrm{min})$, 


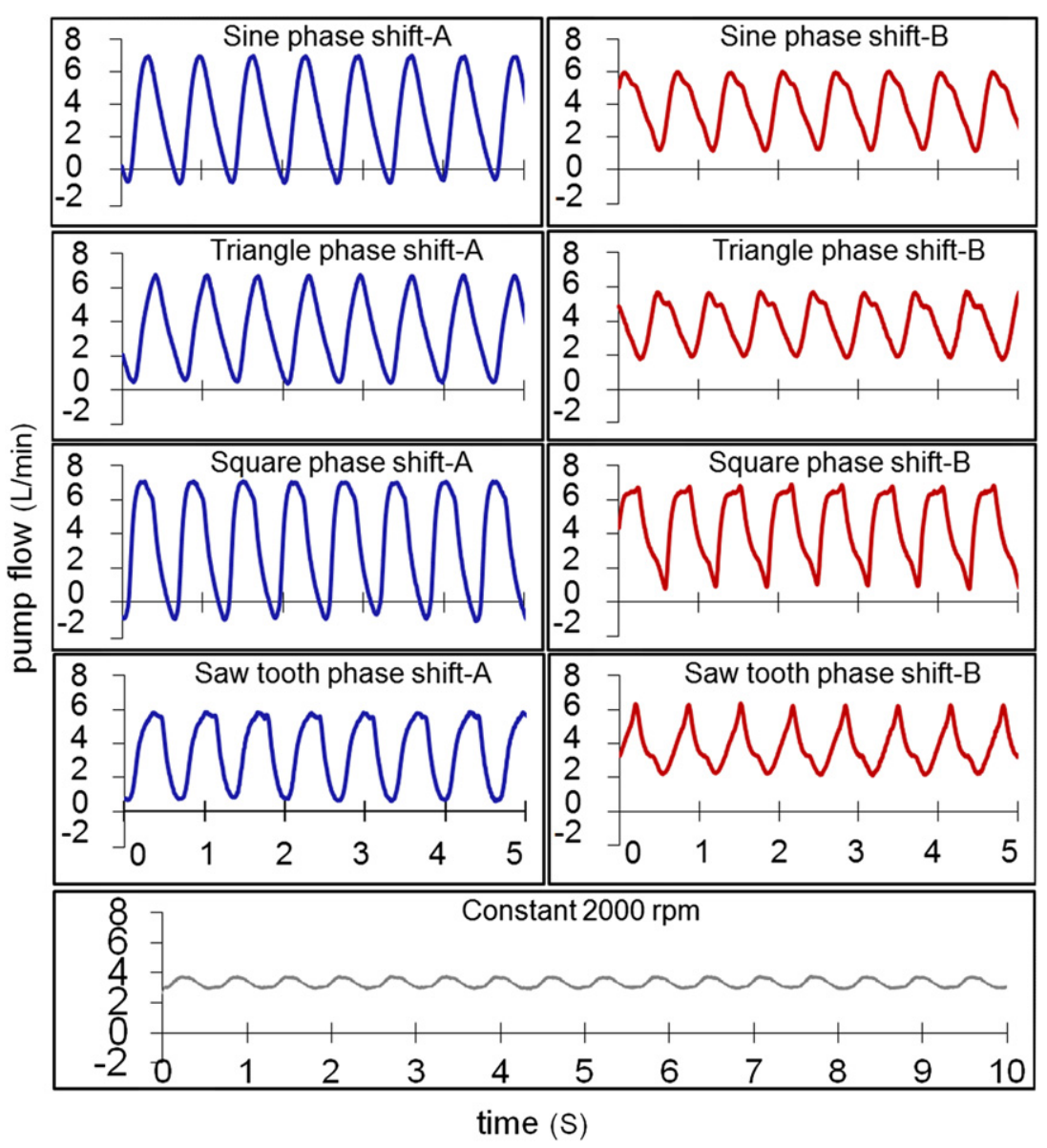

FIGURE 5. Variation of pump flow patterns due to different pump speed.

and sine A $(-0.88 \pm 0.53 \mathrm{~L} / \mathrm{min})$ have negative minimum PF indicating back flow through the pump $(P=.0001)$.

To compare the effect of pump speed patterns on different energetic and hemodynamic parameters, Table 3 lists Tukey's post test results. There is a significant difference between different pump speed patterns in the case of $P$ values less than .05 .

\section{DISCUSSION}

This is the first study to use different basic waveforms to modulate an RBP's speed, synchronized with the ECG, and to investigate its effect on LV unloading and hemodynamics. In the literature, there are few reports describing attempts to modulate the rotary pump's speed, which only focus on asynchronous speed modulation, ${ }^{12}$ mathematical

TABLE 2. Backflow through the pump per each heartbeat

\begin{tabular}{lcccc}
\hline & Square A & Square B & Triangle A & Sine A \\
\hline $\begin{array}{l}\text { Mean backflow } \\
\text { (leakage) volume }\end{array}$ & $3.14 \pm 1.40$ & $0.41 \pm 0.23$ & $0.53 \pm 0.50$ & $1.59 \pm 0.91$ \\
per heartbeat $(\mathrm{mL})$ & & & & \\
\hline
\end{tabular}

modeling, ${ }^{13-15}$ and in vitro investigation of synchronized pump using just sinusoidal waveform. ${ }^{16}$

The presented study is in line with the findings of Maybaum and colleagues, ${ }^{10}$ who demonstrated the importance of the synchrony relationship between the heart and the assist device for ventricular unloading and myocardial recovery. In addition, pump speed modulation using different waveforms with ECG synchronization offers additional control modalities (waveform types and their phase shifts) of hemodynamics. Providing a pulsatile flow together with the other advantages of rotary pumps, such as smaller size, reliable and quiet operation, reduced number of moving components, and higher quality of life for patients can be more beneficial for patients with end-stage heart failure.

From an energy point of view, ventricular SW is defined as the energy consumed by the ventricle to eject blood. Therefore, lower SW means lower work done by ventricle, which results in ventricle unloading. On the other hand, according to the Frank-Starling law of the heart, the lower the $\mathrm{EDV}$, the smaller the SV and the force of contraction during systole, which will in turn also reduce SW. As a result, SW and EDV are linked and both should be considered to evaluate cardiac unloading. Especially for patients with dilated 


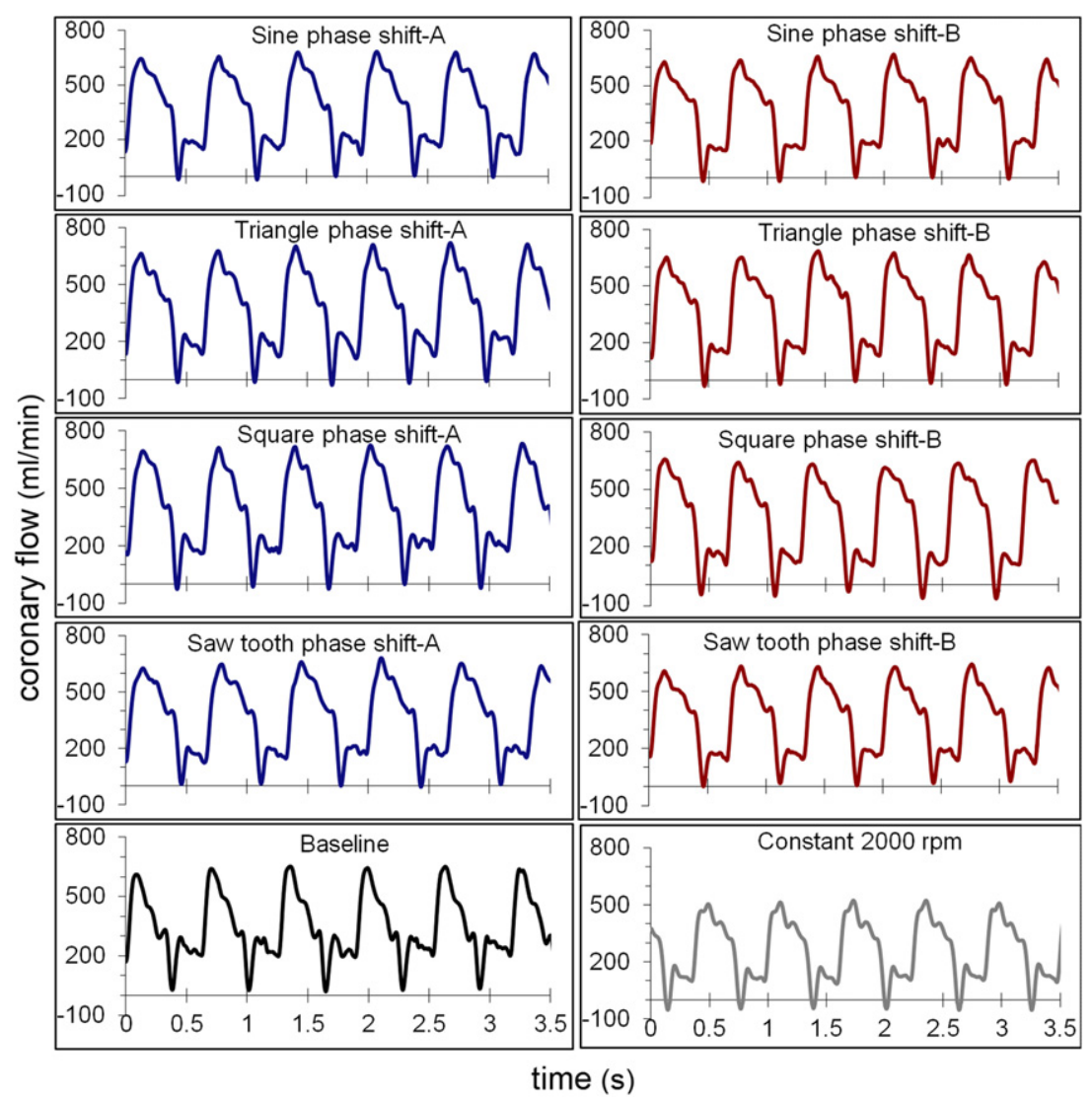

FIGURE 6. Variation of coronary flow $(C F)$ patterns due to different pump speed patterns.

cardiomyopathy, the reduction in EDV is an important determinant of the effectiveness of a therapy, whereas for ischemic disease SW may be preferred because it relates directly to energy consumption required of the viable tissue, and thus also oxygen demand.

By considering the effect of pump speed patterns on LV unloading and hemodynamics, there is a small difference between waveforms with the same phase shift; meanwhile, different phase shifts have a greater effect than waveform type. This is due to a different level of support during systole and diastole by changing the phase shift. The higher pump speed during diastole, the more blood flows through the pump, and as a result the heart needs less work to pump blood. Square B has the highest unloading effect on LV $(69 \%$ of the baseline SW and $68 \%$ of the baseline PVA). On the other hand, it causes back flow $(-0.33 \pm$ $0.94 \mathrm{~L} / \mathrm{min}$ ) through the pump. Backflow through the pump is potentially dangerous for the heart, and therefore its prohibition is an important issue in the selection of the waveform. Triangle B is the second waveform, which has a substantial effect on LV unloading (70\% of the baseline SW and $72 \%$ of the baseline PVA), and the minimum PF $(1.40 \pm 0.66 \mathrm{~L} / \mathrm{min})$ is positive. As a result, it can be considered the best option for LV unloading. In general, for all the waveforms, phase shift B has a more significant effect on hemodynamics and LV unloading than phase shift A, indicating the importance of synchronization between the pump and the heart.

Back flow through the pump is comparable with aortic regurgitation, which is due to incompetence of the aortic valve. This allows some of the blood that was just pumped out of the heart to leak back into it. For the pump used in our study, there is no valve in the inlet and outlet of the pump. As a result, a low level of support may result in backflow during diastole. This is caused by the high pressure difference between the aorta and the LV during diastole and clearly requires a high speed to be overcome. Backflow through the pump, which increases load on heart, is potentially dangerous for a weakened heart, and therefore its prohibition is of importance to the heart, and avoiding back flow (reverse/negative blood flow) through the pump is a critical issue in the selection of the best waveform for modulation of pump speed pattern.

The results show that the pump speed modulation can decrease LV mechanical load, whereas CF remains at a constant level and would not be altered by the pump speed pattern, at least in a healthy heart in which autoregulation determines $\mathrm{CF}$ and it cannot be forced by a pump. This is 


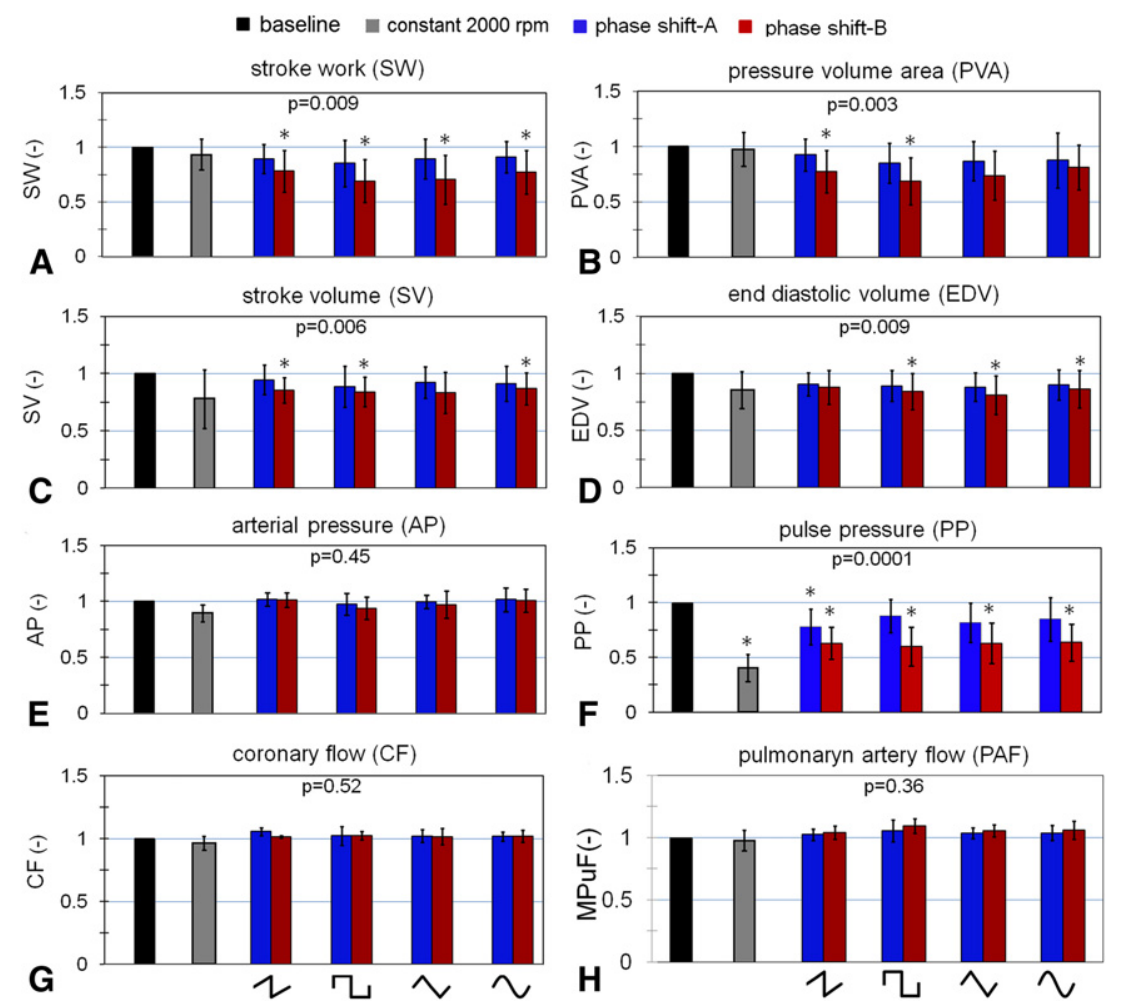

FIGURE 7. A-H, Comparison of different energetic and hemodynamic data due to different applied waveforms to the pump. $* P<.05$ vs baseline.

likely to be beneficial for the myocardium because of the negative impact of reduced myocardial blood flow for heart recovery and device weaning. ${ }^{17}$ In the present study, small
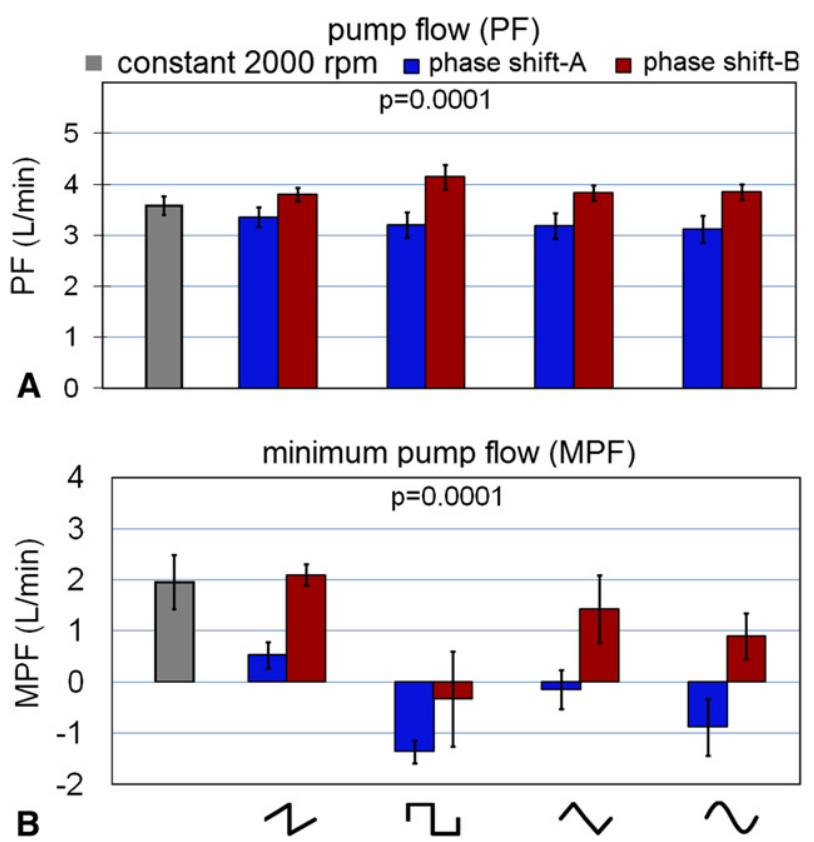

FIGURE 8. Variation of mean pump flow $(P F)$ values $(A)$ and mean minimum PF (B) due to different pump speed patterns. variations in the $\mathrm{CF}$ due to mechanical support of the heart are in contrast with the finding of Maybaum and colleagues ${ }^{10}$ who observed low $\mathrm{CF}$ during support with $\mathrm{VAD}$, which can be the result of a difference between assist devices (positive displacement pump vs rotary pump). In addition, they studied patients with end-stage heart failure, whereas our study is based on healthy sheep.

Blood pressure in the aorta consists of steady and pulsatile components. The steady part, which correlates with the mean AP, is mainly due to the cardiac output and the resistance of the aorta, whereas the pulsatile part, which represents the PP, depends mostly on the compliance of the aorta, ejection ability of the heart, and reflected pressure waves to the heart. This study shows that speed modulation of the pump decreases the PP, whereas the mean AP remains almost at a constant level.

We focus on implantable rotary pumps (or in this case a simulation thereof), and the findings cannot be transferred to cardiopulmonary bypass where long cannula lengths and the oxygenator dampen the effect of pump speed modulation. ${ }^{18}$ We kept the inlet and outlet cannulae short to simulate the implantable devices and avoid energy losses inside them.

Lack of heart failure is a major limitation of this study, although there are no good models available that mimic heart failure as it is seen in typical VAD candidates. The current study does demonstrate the feasibility of pulsing 
TABLE 3. Tukey's test results on the comparison of the pump speed patterns effect on different energetic and hemodynamics parameters

\begin{tabular}{|c|c|c|c|c|c|c|}
\hline \multirow[b]{2}{*}{ Pump speed pattern } & SW & PVA & SV & EDV & $\mathbf{P P}$ & MPF \\
\hline & \multicolumn{6}{|c|}{ Significant? $P<.05$ ? } \\
\hline Sine A vs sine B & No & No & No & No & No & Yes \\
\hline Sine A vs triangle A & No & No & No & No & No & Yes \\
\hline Sine A vs square B & Yes & Yes & No & No & Yes & No \\
\hline Sine A vs saw tooth A & No & No & No & No & No & Yes \\
\hline Sine A vs saw tooth B & No & No & No & No & Yes & No \\
\hline Sine $\mathrm{B}$ vs triangle $\mathrm{A}$ & No & No & No & No & Yes & Yes \\
\hline Sine $B$ vs triangle $B$ & No & No & No & No & No & Yes \\
\hline Sine B vs square A & No & No & No & No & Yes & Yes \\
\hline Sine B vs saw tooth A & No & No & No & No & Yes & Yes \\
\hline Sine B vs saw tooth B & No & No & No & No & No & Yes \\
\hline Triangle A vs triangle B & No & No & No & Yes & Yes & No \\
\hline Triangle A vs square A & No & No & No & No & No & Yes \\
\hline Triangle A vs square $B$ & Yes & Yes & No & No & Yes & Yes \\
\hline Triangle A vs saw tooth B & No & No & Yes & No & Yes & Yes \\
\hline Triangle B vs square A & No & No & No & No & Yes & No \\
\hline Triangle B vs square B & No & No & No & No & No & Yes \\
\hline Triangle B vs saw tooth A & No & No & No & Yes & Yes & No \\
\hline Triangle B vs saw tooth B & No & No & No & No & No & Yes \\
\hline Square A vs square B & No & No & No & No & Yes & Yes \\
\hline Square A vs saw tooth B & No & No & No & No & Yes & No \\
\hline Square B vs saw tooth A & Yes & Yes & No & Yes & Yes & Yes \\
\hline Saw tooth A vs saw tooth B & No & No & No & No & Yes & Yes \\
\hline
\end{tabular}

$S W$, Stroke work; $P V A$, pressure-volume area; $S V$, stroke volume; $E D V$, end-diastolic volume; $P P$, pulse pressure; $M P F$, minimum pump flow.

RBPs and provides a useful initial step toward pulsatile rotary pumps, which may be important for treatment of endstage heart failure. The authors leave the effect of pulsatility on hemolysis and energy consumption of the pump for future studies.

\section{CONCLUSIONS}

Myocardial energy and volume are significantly influenced by the modulation of pump speed. Therefore, this technique is suitable for unloading the supported heart and stimulating myocardial recovery. Furthermore, the pump speed modulation provides pulsatile arterial hemodynamics that might reduce the risk of adverse events caused by a lack of vascular pulsatility, such as gastrointestinal bleeding. Nevertheless, more studies are required to find better strategies for the speed modulation of rotary pumps and to achieve an optimal heart load control to enhance myocardial recovery.

The authors thank the staff of the Experimental Surgery Institute of the University of Bern and Brigitta Gahl for statistics advice.

\section{References}

1. Prosia M, Perktoldb K, Schima H. Effect of continuous arterial blood flow in patients with rotary cardiac assist device on the washout of a stenosis wake in the carotid bifurcation: a computer simulation study. J Biomech. 2007;40:2236-43.

2. Crow S, John R, Boyle A, et al. Gastrointestinal bleeding rates in recipients of nonpulsatile and pulsatile left ventricular assist devices. J Thorac Cardiovasc Surg. 2009;137:208-15.

3. Hayes HM, Dembo LG, Larbalestier R, O’Driscoll G. Management options to treat gastrointestinal bleeding in patients supported on rotary left ventricular assist devices: a single-center experience. Artif Organs. 2010;34:703-6.

4. Nose Y. Nonpulsatile mode of blood flow required for cardiopulmonary bypass and total body perfusion. Artif Organs. 1993;1993:92-102.

5. Undar A. Myths and truths of pulsatile and nonpulsatile perfusion during acute and chronic cardiac support. Artif Organs. 2004;28:439-43.

6. Travis AR, Giridharan GA, Pantalos GM, et al. Vascular pulsatility in patients with a pulsatile- or continuous-flow ventricular assist device. J Thorac Cardiovasc Surg. 2007;133:517-24

7. Hetzer R, Muller J, Weng Y, et al. Cardiac recovery in dilated cardiomyopathy by unloading with a left ventricular assist device. Ann Thorac Surg. 1999;68:742-9.

8. Burkhoff D, Klotz S, Mancini DM. LVAD-induced reverse remodeling: basic and clinical implications for myocardial recovery. J Card Fail. 2006;12:227-39.

9. Barbone A, Holmes JW, Heerdt PM, et al. Comparison of right and left ventricular responses to left ventricular assist device support in patients with severe heart failure: a primary role of mechanical unloading underlying reverse remodeling. Circulation. 2001;104:670-5.

10. Maybaum S, Epstein S, Beniaminovitz A, et al. Partial loading of the left ventricle during mechanical assist device support is associated with improved myocardial function, blood flow and metabolism and increased exercise capacity. J Heart Lung Transplant. 2002;21:446-54.

11. Pan J, Tompkins WJ. A real time QRS detection algorithm. IEEE Trans Biomed Eng. 1985;32:230-6.

12. Bearnson GB, Olsen DB, Khanwilkar PS, et al. Pulsatile operation of a centrifugal ventricular assist device with magnetic bearings. ASAIO J. 1996;42:620-4.

13. Vandenberghe S, Segers P, Meyns B, Verdonck PR. Unloading effect of a rotary blood pump assessed by mathematical modeling. Artif Organs. 2003;27: 1094-101.

14. Cox LGE, Loerakker S, Rutten MCM, et al. A mathematical model to evaluate control strategies for mechanical circulatory support. Artif Organs. 2009;33: 593-603.

15. Pirbodaghi T, Weber A, Carrel C, Vandenberghe S. Effect of pulsatility on the mathematical modeling of rotary blood pumps. Artif Organs. 2011;35:825-32.

16. Vandenberghe S, Segers P, Antaki JF, Meyns B, Verdonck PR. Hemodynamic modes of ventricular assist with a rotary blood pump: continuous, pulsatile, and failure. ASAIO J. 2005;51:711-8.

17. Xydas S, Rosen RS, Pinney S, et al. Reduced myocardial blood flow during left ventricular assist device support: a possible cause of premature bypass graft closure. J Heart Lung Transplant. 2005;24:1976-9.

18. Ündar A, Lodge AJ, Daggett CW, et al. The type of aortic cannula and membrane oxygenator affect the pulsatile waveform morphology produced by a neonateinfant cardiopulmonary bypass system in vivo. Artif Organs. 1998;22:681-6. 